\title{
DOUBLE AORTIC ARCH - A CASE REPORT
}

\author{
Syed Shahid Nafees, Umair Younus, Nasir Ali, Inamullah Khan
}

Armed Forces Institute of Cardiology/National Institute of Heart Disease (AFIC/NIHD)/National University of Medical Sciences (NUMS) Rawalpindi Pakistan

\begin{abstract}
Double Aortic Arch is a rare congenital cardiovascular anomaly. Its first successful surgery was performed by Robert Gross in 1945 at Children Hospital Boston, USA. It accounts for 0.4 to 1\% of all congenital cardiac defects. Patients having a Double Aortic Arch mostly present with symptoms in the 1st week of life but depending upon the severity of symptoms can present at any age in childhood. We present a case report of two month old baby with noisy breathing, intermittent cough, gross jugular notch retraction and sub costal recession. His Cardiac CT was subsequently done which showed a Double Aortic Arch of left dominant variety encircling the trachea. Surgery was done and the encircling artery compressing the trachea was recognized, dissected and interrupted. Marked relief of tracheal and/or esophageal compression was evident from operation day. Post operative recovery was speedy. Patient's follow up of was done at 1,4 and 24 weeks. His recovery was unremarkable. Currently he was thriving well.
\end{abstract}

Keywords: Double aortic arch, Vascular ring.

This is an Open Access article distributed under the terms of the Creative Commons Attribution License (http://creativecommons.org/licenses/by/4.0), which permits unrestricted use, distribution, and reproduction in any medium, provided the original work is properly cited.

\section{INTRODUCTION}

Double Aortic Arch is a congenital vascular anomaly of complete vascular rings ${ }^{1,2}$. It is the most common anomaly involving the Aortic Arch System $^{3}$. It accounts for 0.4 to $1 \%$ of all congenital cardiac defects. Patients having a double aortic arch mostly present with symptoms in the 1st week of life but depending upon the severity of symptoms can present at any age in childhood. Sporadically they have been found in adult patients' too ${ }^{4}$.

Symptoms depend upon the degree of compression of trachea and/or esophagus. Respiratory symptoms include stridor, noisy breathing, repeated pneumonias, wheeze and cough whereas the esophageal symptoms presents as poor feeding, choking after ingestion of milk/ meals, difficulty in eating and vomiting 5 . It may also mimic as asthma ${ }^{1}$. This leads to a failure to thrive and necessitates parents to seek early medical advice.

\section{CASE REPORT}

A two month old baby presented to Pediatric

Correspondence: Dr Syed Shahid Nafees, HoD Peads Cardic Surgery, AFIC/NIHD Rawalpindi Pakistan
Cardiac Surgery Department Armed Forces Institute of Cardiology/National Institute of Heart Diseases (AFIC-NIHD) Rawalpindi. He had noisy breathing, intermittent cough, gross jugular notch retraction and sub costal recession.

On examination, his heart rate was 150/ minute and respiratory rate was $60 / \mathrm{min}$. He weighed $3.5 \mathrm{~kg}$, was pink in color and had $\mathrm{SaO}_{2}$ $98 \%$ in air. Chest auscultation revealed breath sounds with croaking sound on each inspiration and heart auscultation had a harsh pan-systolic murmur at left upper chest. Rest of the general physical, and other systemic examination was unremarkable.

Non-invasive routine investigations and Chest X-ray and ECG were within normal limits.

His 2D Echocardiography done at a private setup in Peshawar revealed small Ventricular Septal Defect (VSD). This finding did not explain his signs and symptoms which were out of proportions of a VSD. His Cardiac CT was subsequently done which showed a Double Aortic Arch of left dominant variety encircling the trachea. Patient was then referred to Paediatric Cardiac Surgery Department AFIC-NIHD for definitive surgery. 


\section{Surgical Technique}

At the Paediatric cardiac surgery department, all the risks and benefits of Double

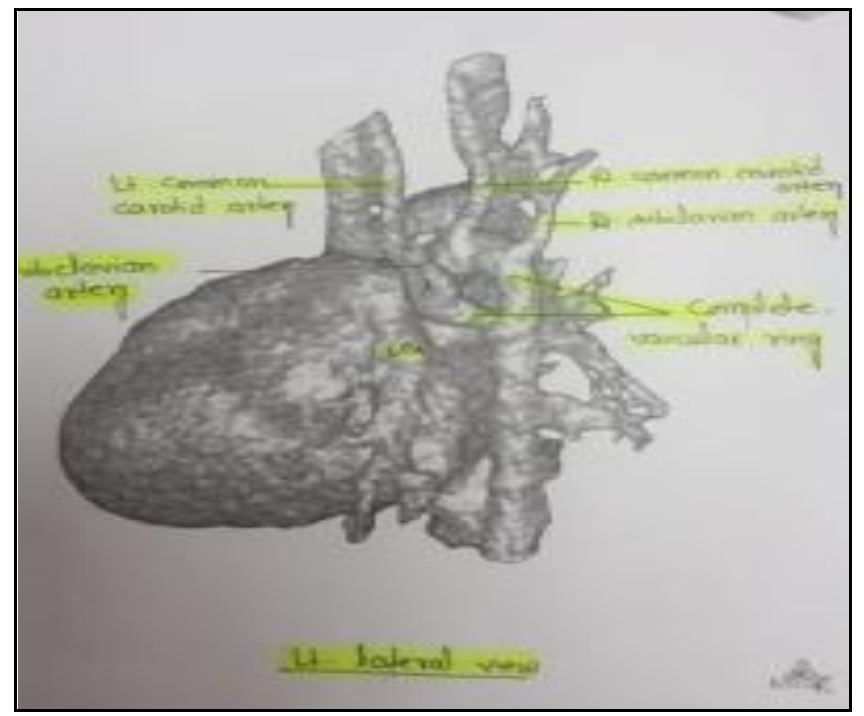

Figure-1: Cardiac CT angiogram (Posterior view of Heart).

Aortic Surgery were explained to the parents and a written high-risk informed consent was obtained from them.

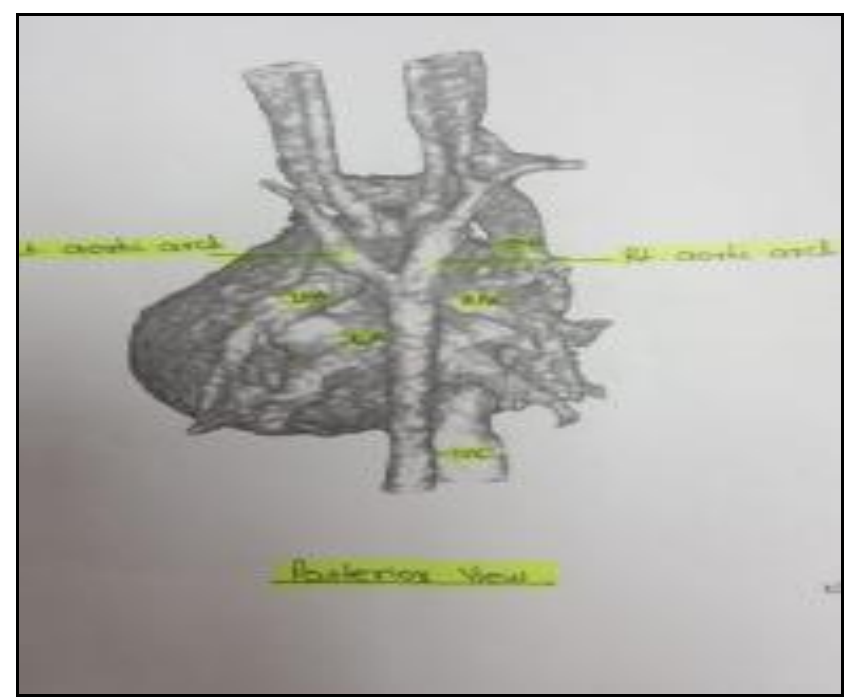

Figure-2: Cardiac CT - angiogram (Lateral view).

After induction of anaesthesia, the patient was placed in left lateral position. Patient was prepared. Right postero-lateral thoracotomy was done and the chest entered through 4 th intercostal space. After dissection and gentle retraction of mediastinal pleura and lung tissue, dissection on the descending aorta, intercostal arteries and neighboring soft tissue was done. The encircling artery which was compressing the

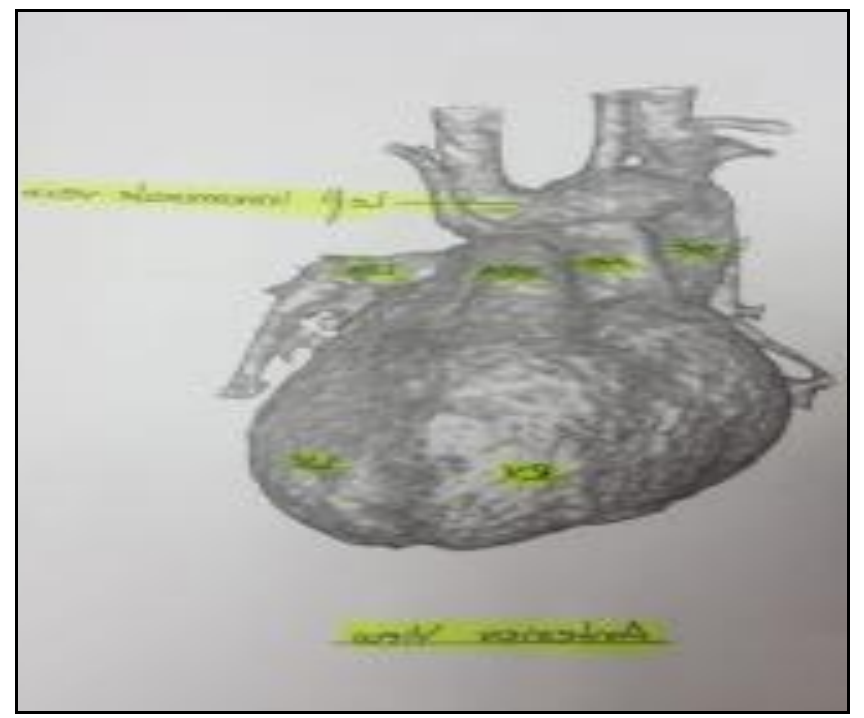

Figure-3: Cardiac CT - angiogram (Anterior View).

trachea was recognized, dissected and interrupted under proximal and distal vascular clamps. Haemostasis was secured. Right pleural drain of size 10 Fr was placed. Local aneasthesia was infiltrated in the 3rd to 5th intercostal space. Ribcage was approximated with rib approximeter and closed with Ethibond 1 suture in an interrupted fashion. Muscles were approximated in two layers and sub cutaneous tissues and skin closed with Vicryl 3/0 suture. Antiseptic dressing was done and patient was shifted to ICU in a hemodynamically stable condition.

After stabilizing arterial blood gases and other anesthetic parameters, the patient was extubated four hours later. Baby now had a normal respiration, his stridor vanished altogether and he no longer had any in-drawing of jugular notch area or sub costal recession.

On $2^{\text {nd }}$ post operative day, he was shifted to high dependency unit and on $5^{\text {th }}$ post operative day he was discharged home, in a symptom-free state.

Patient's follow up of was done at 1,4 and 24 weeks. His recovery was unremarkable. Currently he is thriving well. 


\section{DISCUSSION}

Double Aortic Arch is a rare congenital cardiovascular anomaly. It was first reported in literature in 1737 where by Hommel and the first successful surgery was performed by Robert Gross in 1945 at Children Hospital Boston, USA 6 .

In Pakistan, only a case report of 2 months old female who was born to a cocaine abuser mother has been reported. Her Double Aortic Arch was found compressing trachea and esophagus at autopsy?

Embryologically, Double Aortic Arch is the persistence of both right and left 4 th aortic arches. The ascending aorta, after arising from left ventricle divides into two aortic arches which encircle the trachea and / or esophagus leading to their compression. These two arches subsequently unite with each other to form descending thoracic aorta.

Anatomically, the Double Aortic Arch has the following three sub-types 8 :

Right dominant arch (found in $70 \%$ of cases)

Left dominant arch (found in $25 \%$ of cases)

Co-dominant arch where both arches are of same size (found in $5 \%$ of cases)

From the right aortic arch arise the right carotid and right sub-clavian arteries. It then passes to the right, goes behind the esophagus and joins the left aortic arch.

The left aortic arch gives off left common carotid and left sub-clavian arteries, passes anterior to the trachea, moves to left and joins the right aortic arch.

Cases with mild obstruction present around 2 years of age, while cases of severe compression come to notice at the $1^{\text {st }}$ day of birth. Symptoms depend upon the degree of tracheal and/or esophageal compression which may result in stridor, wheezing, dyspnea at rest, cough, poor feeding, choking after milk ingestion and vomiting. Long standing symptoms may also lead to tracheomalacia
Barium Swallow, Cardiac CT Angiogram ${ }^{9}$ and MRI Heart aid in the definitive diagnosis, but 2D-Echocardiography is not of much help in the diagnostic work up apart from screening other heart lesons.

Asymptomatic patients may never require treatment but severely symptomatic patients, surgery is needed ${ }^{10}$. The principles of surgery are essentially the same as described by Gross. Postero-lateral thoracotomy is the usual surgical approach. Right or left thoracotomy is done on the side contra lateral to the dominant arch (if left arch is dominant, it is right thoracotomy is done and vice versa.) After entering the chest wall, surgical interruption is done. The part of the nondominant arch where it joins its dominant counterpart is interrupted. In the absence of symptoms, a conservative wait-and-watch approach is adopted.

Complications of surgery include bleeding, recurrent laryngeal or phrenic nerve neuropraxia or permanent damage which may lead to vocal cord or hemi-diaphragm paralysis. Excessive retraction of lung tissue may lead to haematoma. Dissection and damage around thoracic duct may lead to post-operative chlyothorax.

Marked relief of tracheal and/or esophageal compression is evident from operation day. Post operative recovery is speedy. Follow up is done at 3 monthly intervals for one year and then 6 monthly for next 2-3 years.

\section{CONCLUSION}

This anomaly is very rare and is easily liable to be missed by the clinician in the first or second visit of the baby. A very high index of suspicion with early referral to paeds cardiologist who in turn recommends early CT-angio is the only confirmed way of its diagnosis. Once diagnosed it is corrected surgically with a very high cure rate.

\section{CONFLICT OF INTEREST}

This study has no conflict of interest to be declared by any author. 


\section{REFERENCES}

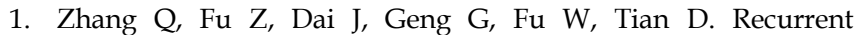
Wheezing and Cough Caused by Double Aortic Arch, Not Asthma. Case Rep Cardiol 2017; 2017: 8079851.

2. Wafy. "Vascular ring", the hidden congenital anomaly having symptoms that may mimic asthma: A case report [Internet]. [cited 2019]. Available from: http:/ / www.ejbronchology.eg.net/ article.asp?issn=1687-8426; year $=2016$; volume $=10$;issue $=1$; spage $=69$; epage $=72$;aulast $=$ Wafy

3. Ullmann $\mathrm{N}$, Menchini $\mathrm{L}$, Salerno $\mathrm{T}$, Tomà $\mathrm{P}$, Cutrera R. Late diagnosis of double aortic arch: consequences on long-term follow-up. Pediatr Pulmonol 2014; 49(3): e75-77.

4. Yilmaz M, Tok M, Cengiz M. Asymptomatic balanced-type double aortic arch in an elderly patient: a case report. Heart Surg Forum 2007; 10(4): e297-98.
5. Calabrese C, Corcione N, Di Spirito V, Guarino C, Rossi G, Domenico GG, et al. Recurrent respiratory infections caused by a double aortic arch: The diagnostic role of spirometry. Respir Med Case Rep 2013; 8(1): 47-50.

6. Wolman IJ. Syndrome of constricting double aortic arch in infancy: Report of a case. J Pediatr 1939; 14(4): 527-33.

7. Afzal MN. Fatal double aortic arch anomaly and maternal cocaine abuse. J Coll Physicians Surg Pak 2003; 13(3): 166-67.

8. Santos FCGB, Croti UA, Marchi CHD, Hassem SS. Double aortic arch associated with pulmonary atresia with ventricular septal defect. Braz J Cardiovasc Surg 2016; 31(1): 1-5.

9. Hanneman K, Newman B, Chan F. Congenital variants and anomalies of the aortic arch. Radio Graph 2016; 37(1): 32-51.

10. Berge TM, van der Laag J, van der Ent CK, Beek FJA. Clinical, radiological and functional follow-up after surgical decompression of double aortic arch. Pediatr Radiol 2002; 32(8): 561-66. 